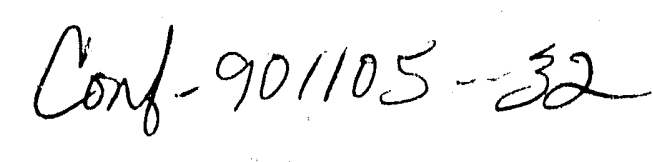

\title{
X-RAY REFLECTIVITY MEASUREMENTS OF SURFACE ROUGHNESS USING ENERGY DISPERSIVE DETECIION
}

SAND $-90-1787 \mathrm{C}$

E. CHASON AND D.T. WARWICK

DE9 004819

Sandia National Laboratories, Albuquerque, NM 87185

\begin{abstract}
We describe a new technique for measuring $X$-ray reflectivity using energy dispersive $\mathrm{X}$-ray detection. The benefits of this method are the use of a fixed scattering angle and parallel detection of all energies simultaneously. These advantages make the technique more readily useable with laboratory $\mathrm{X}$-ray sources and more compatible with growth chambers. We find excllent agreement between the calculated Fresnel reflectivity and the reflectivity obtained from a smooth $\mathrm{Ge}(001)$ surface. Reflectivities obtained during $500 \mathrm{eV} \mathrm{Xe}$ ion bombardment of Ge surfaces demonstrate the sensitivity of the technique to be better than $1 \AA$.
\end{abstract}

\section{INTRODUCTION}

As the need for atomic-level control of interfaces in advanced materials preparation grows, the need for measurement of interface roughness in this regime becomes increasingly important. X-ray reflectivity is a non-destructive, in situ probe of surface roughness over the region of $\sim 1-50 \mathrm{~A}$. The high penetrability of $\mathrm{X}$-ray radiation is a further advantage of the technique, making it applicable in gaseous environments (such as chemical vapor deposition (CVD) reactors) where electron diffraction is not possible.

Widespread use of X-ray reflectivity as a surface roughness diagnostic has been hampered by the experimental difficulties associated with it. The large dynamic range required (typically $>10^{5}$ ) makes it difficult to achieve on a standard laboratory X-ray generator. Advances in the use of X-ray reflectivity as a measure of interface and surface roughness [1-4] and multilayer quality [5] in recent years have been led in large part by experiments performed on synchrotron light sources, although such experiments have also been performed on conventional X-ray systems $[6,7]$. The suitability of X-ray reflectivity as a probe of atomic scale roughness has led us to pursue a laboratory-based alternative to the conventional technique which decreases acquisition time and increases applicability as an in situ, real-time measurement.

In this work, we describe a new approach to measuring X-ray reflectivity using energy dispersive detection. This technique determines the reflectivity as a function of energy at a fixed angle. By working at fixed angle, we lessen the experimental difficulty of scanning small angles at high precision and make the technique more adaptuble for use as an in situ monitor in growth chambers and other situations where angle scanning is difficult. By using an energy dispersive detector, we collect all the scattered radiation in parallel thereby significantly decreasing the data acquisition time $(<1000 \mathrm{~s})$ and making the technique more amenable to laboratory-based X-ray generators.

\section{ORIGIN OF X-RAY REFLECTIVITY}

The relationship between $\mathrm{X}$-ray reflectivity and surface roughness has been treated 


\section{DISCLAIMER}

This report was prepared as an account of work sponsored by an agency of the United States Government. Neither the United States Government nor any agency thereof, nor any of their employees, makes any warranty, express or implied, or assumes any legal liability or responsibility for the accuracy, completeness, or usefulness of any information, apparatus, product, or process disclosed, or represents that its use would not infringe privately owned rights. Reference herein to any specific commercial product, process, or service by trade name, trademark, manufacturer, or otherwise does not necessarily constitute or imply its endorsement, recom. mendation, or favoring by the United States Government or any agency thereof. The views and opinions of authors expressed herein do not necessarily state or reflect those of the United States Government or any agency thereof. 
completely elsewhere, both in terms of diffraction [1] and multi-layer formalisms [8]. Therefore, only the most important points will be repeated here. The origin of X-ray reflectivity is in the index of refraction, $n$, given by the following equation:

$$
n=1 \cdot(h c / E)^{2} N_{e l} r_{0} / 2 \pi
$$

where $E$ is the $X$-ray energy, $N_{e l}$ is the electron density in the bulk and $r_{0}$ is the classical electron radius. Since $\mathbf{n}<1$, there exists a critical angle below which there is total external reflection. Above the critical angle, the reflectivity is given by the Fresnel equations [1] for a perfectly sharp interface. For the range of scattering vectors used in this work, the Fresnel refelctivity $\left(R_{\mathbb{F}}\right)$ may be approximated by:

$$
\mathrm{R}_{\mathrm{F}}(\mathrm{q}) \approx\left(\mathrm{q} / \mathrm{q}_{\mathrm{e}}\right)^{4}
$$

where $q$ is the scattering vector $(q=4 \pi(E / h c) \sin \theta)$, and $q_{c} \approx 4\left(N_{e l} r_{0} \pi\right)^{1 / 2}$. For an interface that is not perfectly abrupt, we define $N(z)$ as the electron density profile in the direction $z$ normal to the sample surface. $N(z)$ has the asymptotic values $N_{e l}$ in the bulk and zero in the vacuum. The variation of the electron density in the transition region from bulk to vacuum determines the q-dependence of the reflectivity. It is shown in ref. [1] that the reflected intensity $(\mathrm{I}(\mathrm{q}))$ is given by:

$$
I(q)=I_{0}(E) R_{r}(q)\left|\int d N / d z \exp (i q z) d z\right|^{2}
$$

where $\mathrm{I}_{0}(\mathrm{E})$ is the incident intensity at energy $\mathrm{E}$. If we normalize the scattered intensity by the Fresnel reflectivity, the ratio is proportional to the fourier tranform of $d N / d z$. At $q=0$, the fourier transform always has the same value $\left(\mathrm{N}_{\mathrm{el}}\right)$ independent of the surface roughness.

\section{EXPERIMENT}

The reflectivity is obtained experimentally by measuring the reflected intensity as a function of the scattering vector. In conventional reflectivity measurements, the X-ray energy is kept constant and $q$ is scanned by changing the scattering angle $\theta$. In the energy-dispersive technique, however, $q$ is scanned by keeping the scattering angle fixed (at $1 \mathrm{deg}$ ) and measuring the reflerted intensity as a function of energy.

The experimental apparatus is shown in figure 1. A broad band of X-ray energy in the range of $5-30 \mathrm{keV}$ is obtained from the Bremsstrahlung radiation produced by a $\mathrm{W}$ anode operated at $30 \mathrm{kV}$ and $100 \mathrm{~mA}$ provides. The energy of the reflected radiation is determined by an intrivsic Ge detector in which a voltage pulse is generated proportional to the energy of the incoming $X$ ray with an energy resolution of approximately $300 \mathrm{eV}$ in the energy range used. The spectrum of reflected energies is acquired in a multichannel analyzer. The slits define a beam divergence of approximately $0.2 \mathrm{deg}$. The $\mathrm{X}$-ray filament is in the line focus mode producing an effective source size 50 microns wide and $1 \mathrm{~cm}$ high.

The sample is $1 \mathrm{~cm} \times 1 \mathrm{~cm} \times 0.1 \mathrm{~cm}$ Ge prepared by pad polishing in Br:methanol. The sample is held in a small vacuum chamber (base pressure $\sim 1 \times 10^{-3}$ torr) mounted on a four-circle Huber goniometer. 10 mil Be windows allow the X-ray beam in and out of the chamber. The vacuum chamber is equipped with a reflection high energy electron diffraction (RHEED) system for independent determination of the quality of the sample surface. The direction of the RHEED beam is perpendicular to the X-ray beam so that both measurments can be made without moving the sample. The sample is mounted on a motorized manipulator for alignment purposes, with an attached $e^{-}$beam heater for sample annealing. A $1 \mathrm{~cm}$, low energy Kaufmann-type ion source is used for sample 


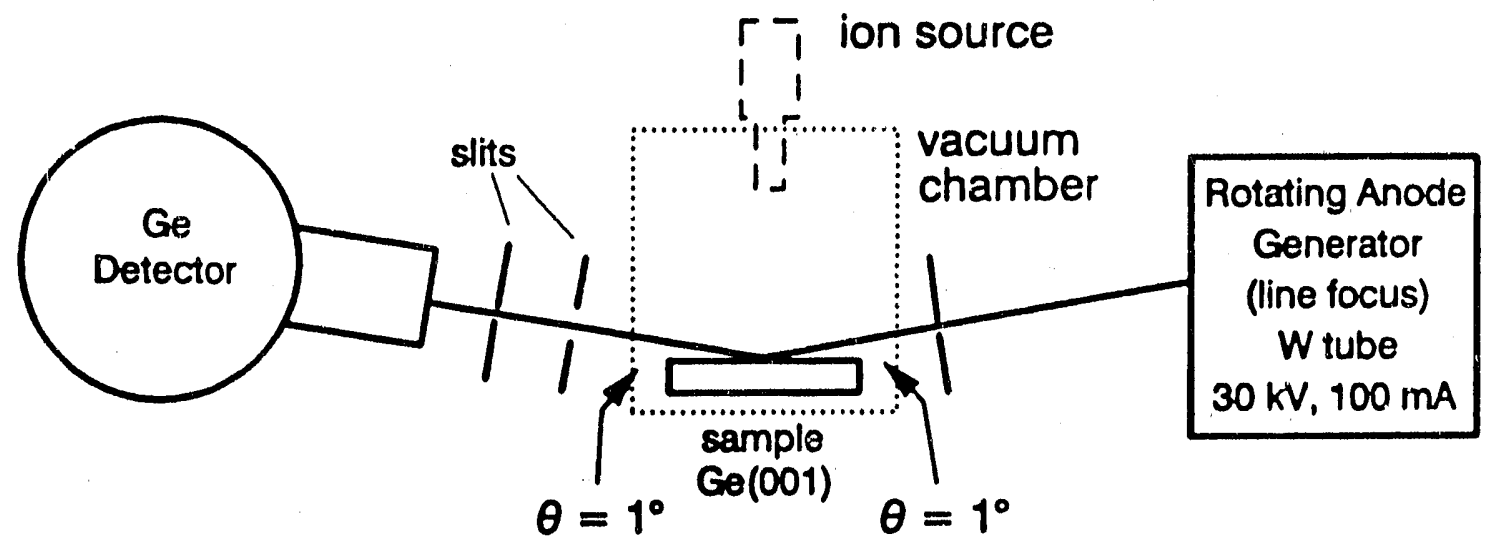

Figure 1. Schematic of $X$-ray reflectometer using energy dispersive detection.

preparation and surface roughening; the incident beam is oriented $45^{\circ}$ to the sample normal,. It is important to note that the vacuum chamber is not required for the reflectivity measurement; it is necessary, however, for surface preparation, RHEED characterization of the surface and controlled surface roughening.

\section{MEASURED SCATTERING FROM GE AND FRESNEL REFLECTIVITY}

The reflected spectrum obtained from a Ge sample which has been repeatedly sputter-cleaned and annealed is shown in figure 2 (solid line). This surface was determined by reflection high energy electron diffraction (RHEED) to be atomically smooth from the presence of a strong specular spot in the "out-of-phase" diffraction condition, well defined Kikuchi bands and three sharp Laue rings; RHEED features indicative of multi-level roughness ("spotty" patterns) were not observed. For comparison, the product of the incident energy spectrum and the Fresnel reflectivity, which is equal to the scattering from a perfectly sharp interface, is shown as the filled circles in figure 2. The scales of the two curves have been adjusted to give the best agreement. The incident energy spectrum is obtained by measuring the scattered intensity in a $\theta-2 \theta$ scan of a graphite crystal and correcting for sample absorption in the reflection geometry (see Cullity [9]). The calculated spectrum is in good agreement with the experimentally obtained one.

The peaks in the spectra at 7.4, 8.4 and $9.8 \mathrm{keV}$ are from the W L-series emission lines; they are present in the incident energy spectrum and do not represent peaks in the reflectivity. The peak in the spectrum from the smooth Ge surface at $11.3 \mathrm{keV}$ is most likely from $\mathrm{Ge} \mathrm{KB}$ fluorescence and thus is not found in the spectrum measured with the graphite crystal. The downturn in the spectrum from the Ge below $7 \mathrm{keV}$ is presumably caused by absorption of the X-rays in the $\mathrm{Be}$ windows. The critical energy for total external reflection from the Ge sample at $\theta=1 \mathrm{deg}$ is $2.5 \mathrm{keV}$, so $\mathrm{q}>\mathrm{q}_{\mathrm{c}}$ for all the measurements discussed in this paper. The deviation of the measured Ge spectrum from the calculated Fresnel spectrum at high energy may be the result of residual surface roughness. However, the agreement in the two spectra is sufficiently good that the spectrum measured from the smooth surface is used to normalize subsequent measured spectra. 


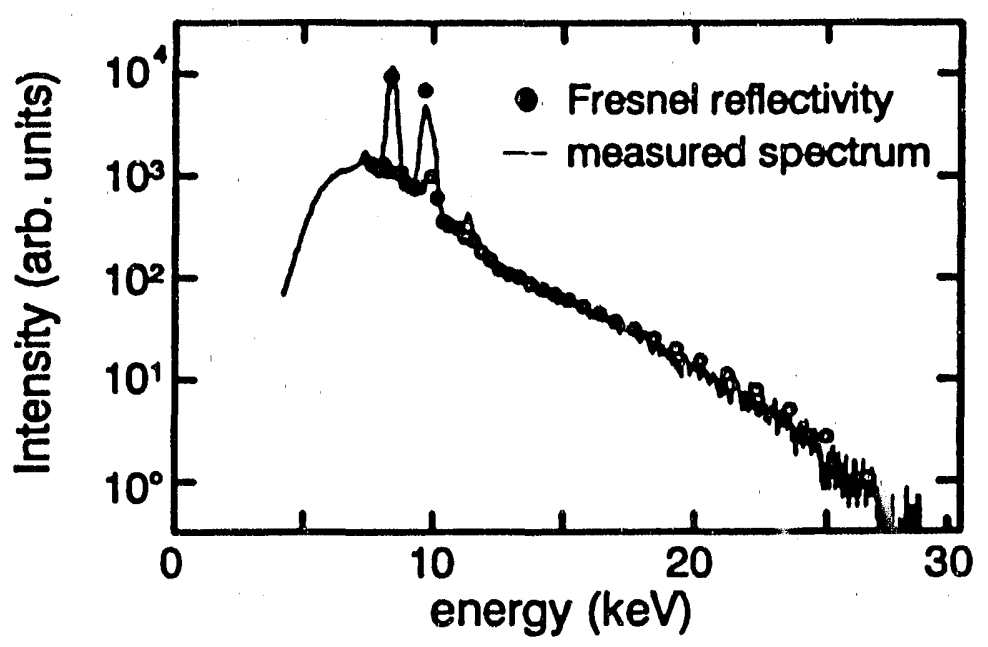

Figure 2. Comparison of reflected spectrum from smooth $\mathrm{Ge}(001)$ surface and Fresnel reflectivity calculated from the incident energy spectrum (circles).

\section{REFLECTIVITY FROM ION-BEAM ROUGHENED Ge SURFACES}

A difficulty in determining the resolution of this technique comes from the lack of samples of known roughness in the 1 - $50 \AA$ regime. Therefore, we produce our own rough surfaces by sputiering the Ge surface at $350^{\circ} \mathrm{C}$ with $500 \mathrm{eV}$ Xe ions at an estimated flux of $100 \mathrm{nA} / \mathrm{cm}^{2}$ (obtained from beam currents measured on a target with no correction for secondary electron emission). This approach has the advantage of not having to change and realign samples between measurements. After the spectrum from the smooth starting surface is obtained, the ion gun is turned on and spectra are measured repeatedly every $1000 \mathrm{~s}$.

A representative series of reflectivity measurements made during the ion-beam roughening is shown in figure 3 . The total sputtering time is shown next to each spectrum. The X-ray reflectivity is normalized to the Fresnel reflectivity, and plotted as a a function of scattering vector. As the sputtering time increases, the width of the reflectivity curve in q-space becomes sharper corresponding to the broadening of the interface width in real-space.

In order to quantitatively determine the change in the interface width during sputtering, we fit the measured spectra to the following form:

$$
\left|(1-A)+A(1-i q \sigma) /\left(1+(q \sigma)^{2}\right)\right|^{2} .
$$

We emphasize that this data analysis is only preliminary; the fitting form was chosen for its empirical agreement with the data but was not derived from a model of the sputtering process. The constant is different for each fit, but the form of the function is chosen so that the value at $q=0$ is always unity, as discussed in section 3 . The fit is shown as the dotted line in figure 3 . Although the form of the density can not be determined uniquely from inversion of the fourier transform, this fitting form is consistent with a density profile given by:

$$
\begin{array}{cl}
N(z)=N_{\mathrm{el}}(1-A \exp (-z / \sigma)) & z>0 \\
0 & z<0
\end{array}
$$

The discontinuity at $z=0$ appears to be a real feature of the data. Attempts to fit the measured spectra without the constant term (1-A) in eq. 4 were not successful.

The value of $\sigma$ obtained from the fitting procedure is shown in figure 4 as a 


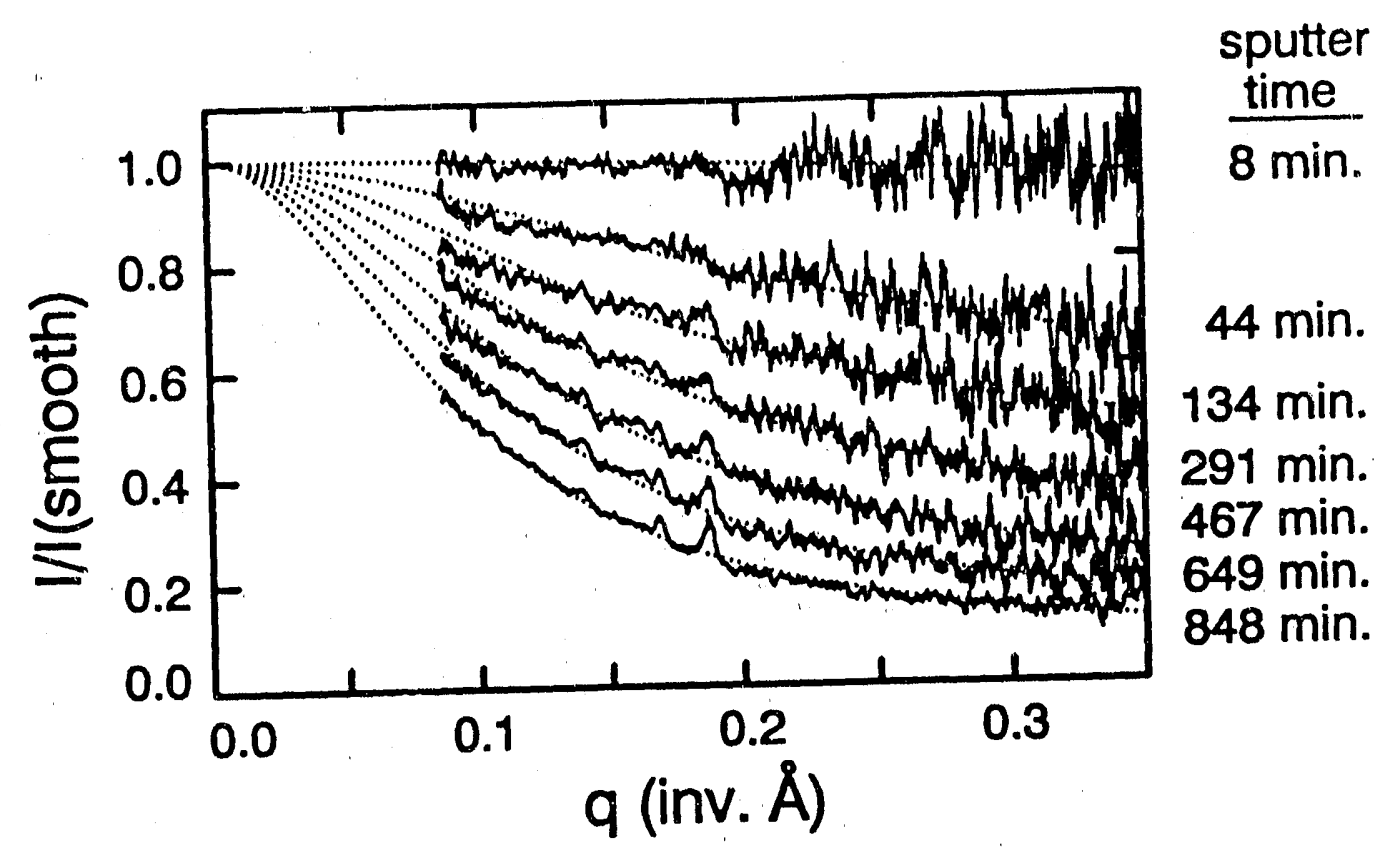

Figure 3. Measured $X$-ray reflectivity, normalized to Fresnel reflectivity from a Ge (001) surface during $500 \mathrm{eV}$ Xe ion bombardment. The total sputtering time is indicated next to each curve. Ion flux is estimated to be $0.1 \mu \mathrm{Narn}{ }^{2}$. Sample temperature is $350^{\circ} \mathrm{C}$. Dotted lines represent fit to the form given in eq. (4).

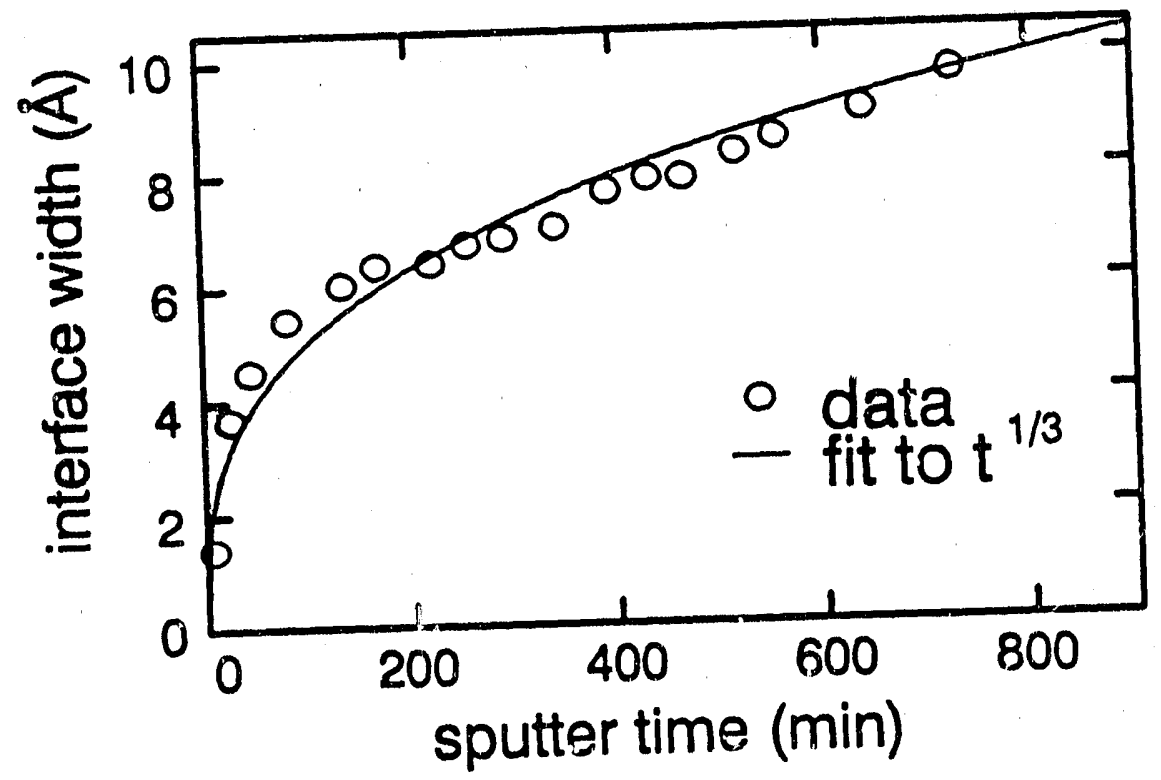

Figure 4. Evolution of interface width during $500 \mathrm{eV}$ Xe ion bombardment of $\mathrm{Ge}(001)$ surface. Values of the interface width are obtained from fitting measured reflectivities to eq. (4). 
function of sputtering time. The solid line on the curve represents a fit to the form $\mathrm{At}^{1 / 3}$ with $A=1.10$; this form is empirical as well. The very rapid roughening at the early stages of sputtering may indicate a transient in the startup of the ion gun (perhaps impurities collected on the gun filament) or the presence of some easily sputtered adsorbate on the Ge surface. Using a multilayer formalism, we hope to develop a more complete analysis of the reflectivity to determine the surface density profile more exactly. The current measurements are presented primarily to demonstrate the sensitivity of the energydispersive reflectivity technique. From the scatter in the data, and the ability to resolve changes in the reflected spectra, we estimate that the sensitivity of the energy-dispersive reflectivity measurement is better than $1 \mathrm{~A}$.

\section{CONCLUSION}

We have developed a new technique for measuring X-ray reflectivity using energydispersive detection. The primary benefits of this technique are measuring reflectivity without changing scattering angle and simultaneous measurement of the entire reflected spectrum. These improvements make the technique more compatible with growth chambers and easier to achieve with laboratory-based X-ray sources. We have demonstrated that the reflectivity from a sputtered and annealed Ge sample agrees with the Fresnel reflectivity calculated from the incident energy spectrum. We have measured the evolution of roughness during $500 \mathrm{eV} \mathrm{Xe}$ sputtering of the Ge surface, demonstrating that this technique can be used as a real-time in situ monitor of surface roughness.

\section{ACKNOWLEDGMENTS}

We gratefully acknowledge the technical assistance of Bob Chesebrough and useful discussion with Barney Doyle. This work was supported by the U.S. Department of Energy under contract DE-AC04-76DP00789.

\section{REFERENCES}

1. J. Als-Nielsen, in Structure and Dynamics of Surfaces, W. Schommers and P. von Blanckenhagen eds, (Springer-Verlag, Berlin, 1986) Chap. 5, p. 181.

2. A. Braslau, P.S. Pershan, G. Swislow, B.M. Ocko and J. Als-Nielsen, Phys. Rev A38, 2457 (1988). A. Braslau, M. Deutsch, P.S. Pershan, A.H. Weiss, J. Als-Nielsen and J. Bohr, Phys. Rev. Lett. 54, 114 (1985).

3. D. Gibbs, B.M. Ocko, D.M. Zehner and S.G.J. Mochrie, Phys. Rev. B38, 7303 (1988).

4. E. Vlieg, A.W. Denier van der Gon, J.F. van der Veen, J.E. MacDonald and C. Norris, Phys. Rev. Lett. 61, 2241 (1988).

5. J.H. Underwood and T.W. Barbee, Appl. Optics 20, 3027 (1981).

6. F.E. Fernandez, C.M. Falco, P. Dhez, A. Khandar-Shahabad, L. Nevot, B. Pardo, J. Corno and B. Vidal, Appl. Phys. Lett. 51, 880 (1987).

7. J.M. Baribeau, Appl. Phys. Lett. 57, 1748 (1990).

8. L.G. Parratt, Phys. Rev. 25, 359 (1954).

9. B.D. Cullity, Elements of X-Ray Diffraction (Addison-Wesley, Reading, Ma, 1978) p. 135. 

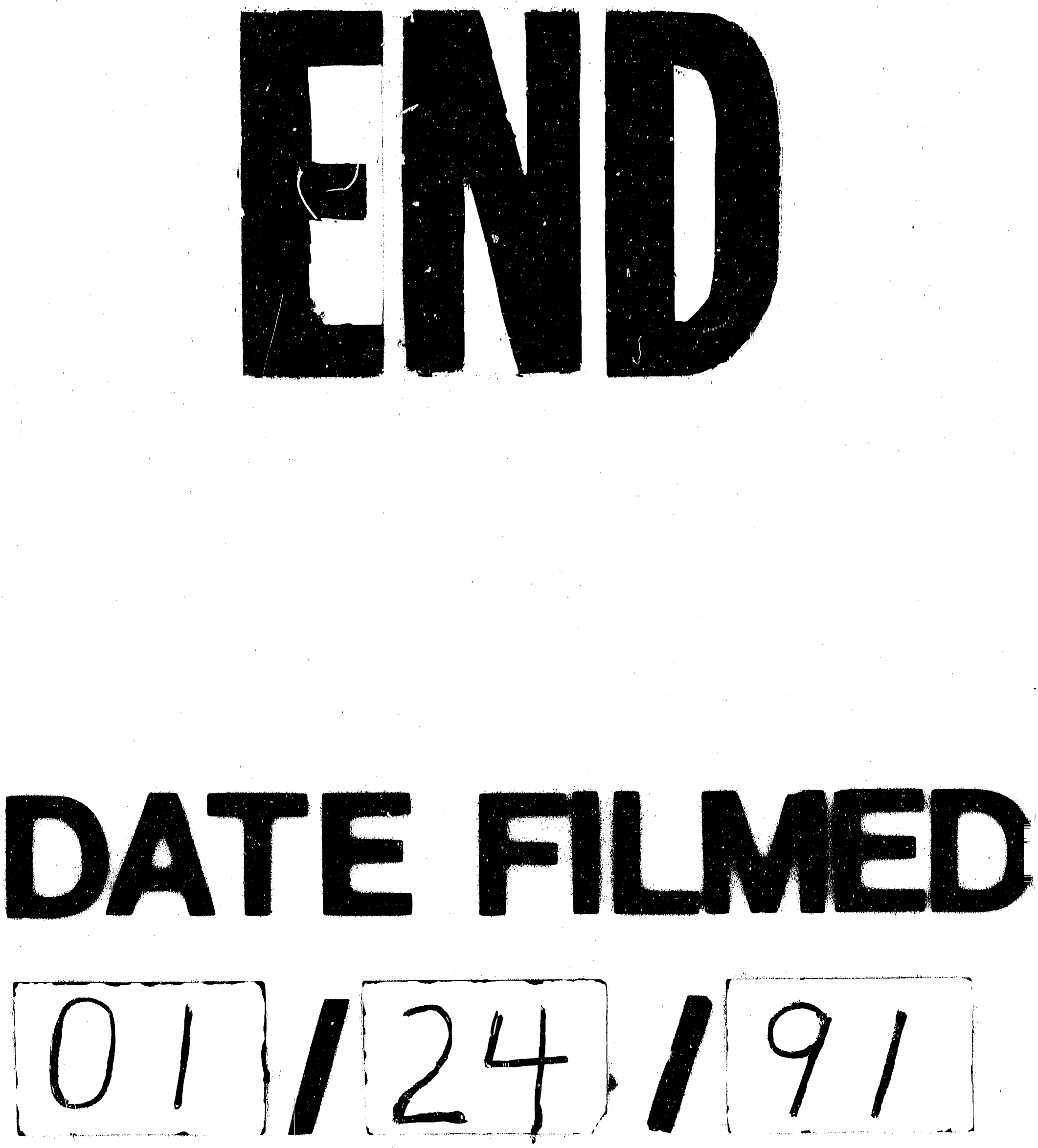
$x_{-1}$ 